\title{
Erratum: $N_{f}=2+1$ QCD thermodynamics with gradient flow using two-loop matching coefficients [Phys. Rev. D 102, 014510 (2020)]
}

Yusuke Taniguchi, Shinji Ejiri, Kazuyuki Kanaya๑, Masakiyo Kitazawa, Hiroshi Suzuki, and Takashi Umeda

$$
\text { (WHOT-QCD Collaboration) }
$$

(Received 11 September 2020; published 28 September 2020)

DOI: 10.1103/PhysRevD.102.059903

In Sec. II C, Eqs. (50) and (51) for "linear + log fit" should read

$$
\langle\mathcal{O}(t, a)\rangle=\langle\mathcal{O}\rangle+t S_{\mathcal{O}}+\frac{Q_{\mathcal{O}}}{\log ^{2}\left(\sqrt{8 t} \Lambda_{\overline{\mathrm{MS}}}\right)}
$$

and

$$
\langle\mathcal{O}(t, a)\rangle=\langle\mathcal{O}\rangle+t S_{\mathcal{O}}+\frac{Q_{\mathcal{O}}^{\prime}}{\log ^{3}\left(\sqrt{8 t} \Lambda_{\overline{\mathrm{MS}}}\right)},
$$

respectively, where $\Lambda_{\overline{\mathrm{MS}}}=332(17) \mathrm{MeV}$ is the QCD lambda parameter for three-flavor QCD quoted in the Particle Data Group [1].

The dotted curves for the linear + log fit in Figs. 2-7, 10, and 12 were showing the results of nonlinear fit by error. The correct figures are given in this Erratum. The caption of Fig. 10 is slightly modified to quote Eq. (51).

Analyses in the paper have been done adopting the above correct formulas, and thus the results and the tables as well as other figures are unchanged.

Published by the American Physical Society under the terms of the Creative Commons Attribution 4.0 International license. Further distribution of this work must maintain attribution to the author(s) and the published articles title, journal citation, and DOI. 

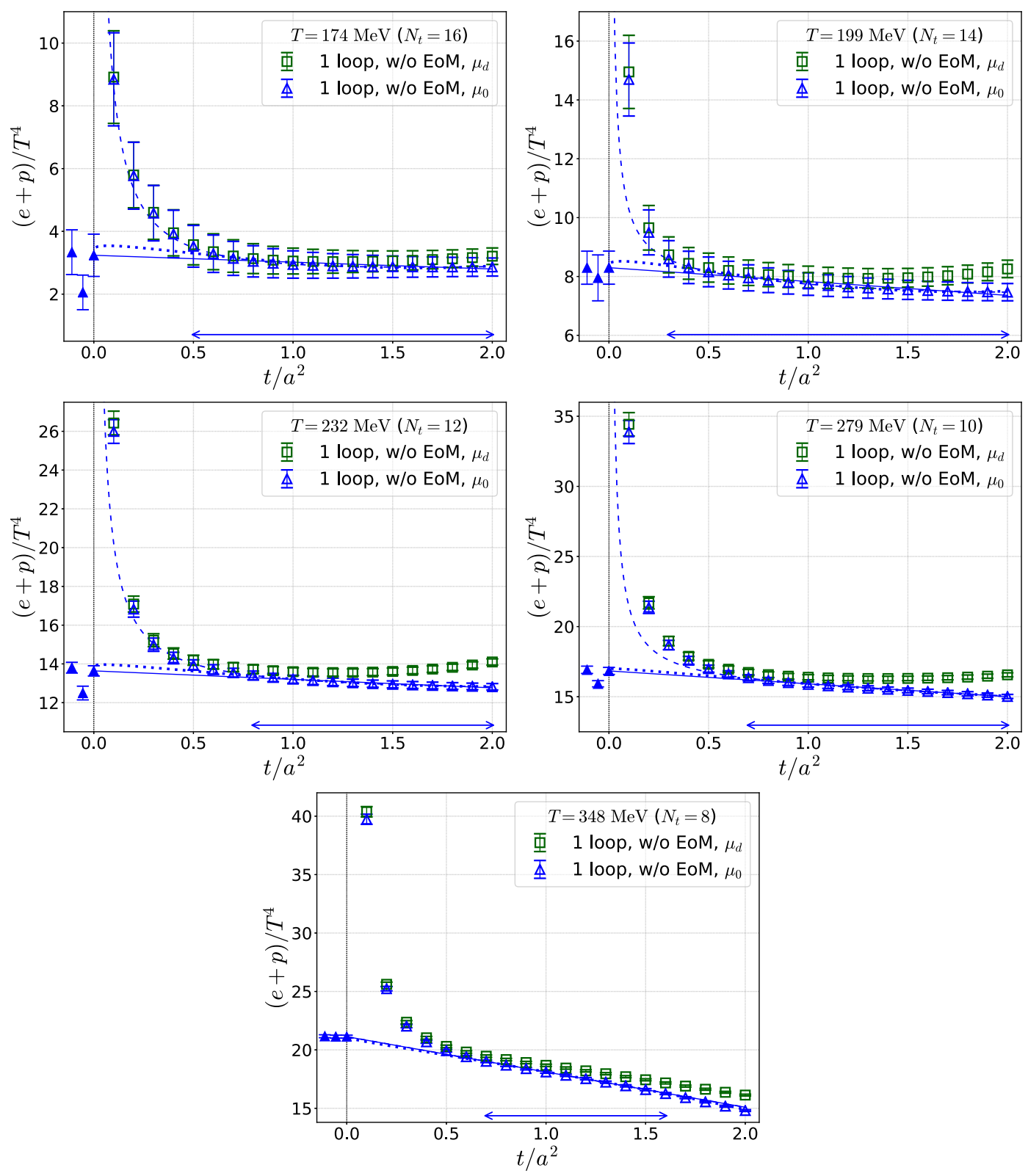

FIG. 2. Entropy density $(\epsilon+p) / T^{4}$ with $\mu_{0}$ (blue) and $\mu_{d}$ (green) scales as a function of the flow time. One-loop matching coefficients of Ref. [10] are used. Also shown are the results of the $t \rightarrow 0$ extrapolations using the data with the $\mu_{0}$ scale: Solid line is the linear fit using the linear window indicated by the arrow at the bottom of each plot, and the symbol at $t / a^{2}=0$ is the result of the linear fit for the physical entropy density in the $t \rightarrow 0$ limit. Fit results with the nonlinear ansatz (49) and linear $+\log$ ansatz (50) are shown by dashed and dotted curves together with the symbols at $t / a^{2}<0$ to the right and to the left, respectively. Errors are statistical only. 

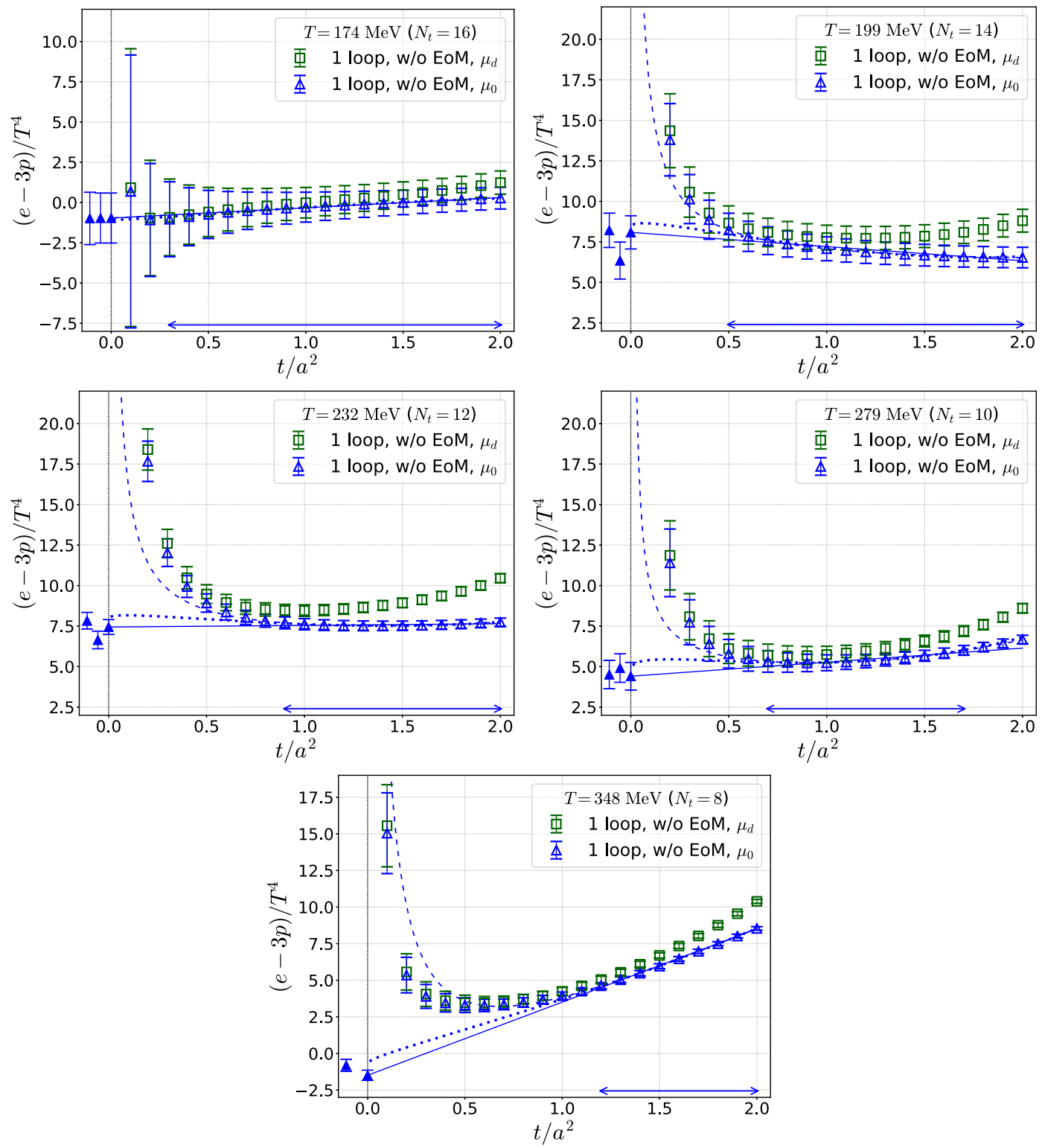

FIG. 3. The same as Fig. 2 but for the trace anomaly $(\epsilon-3 p) / T^{4}$. 

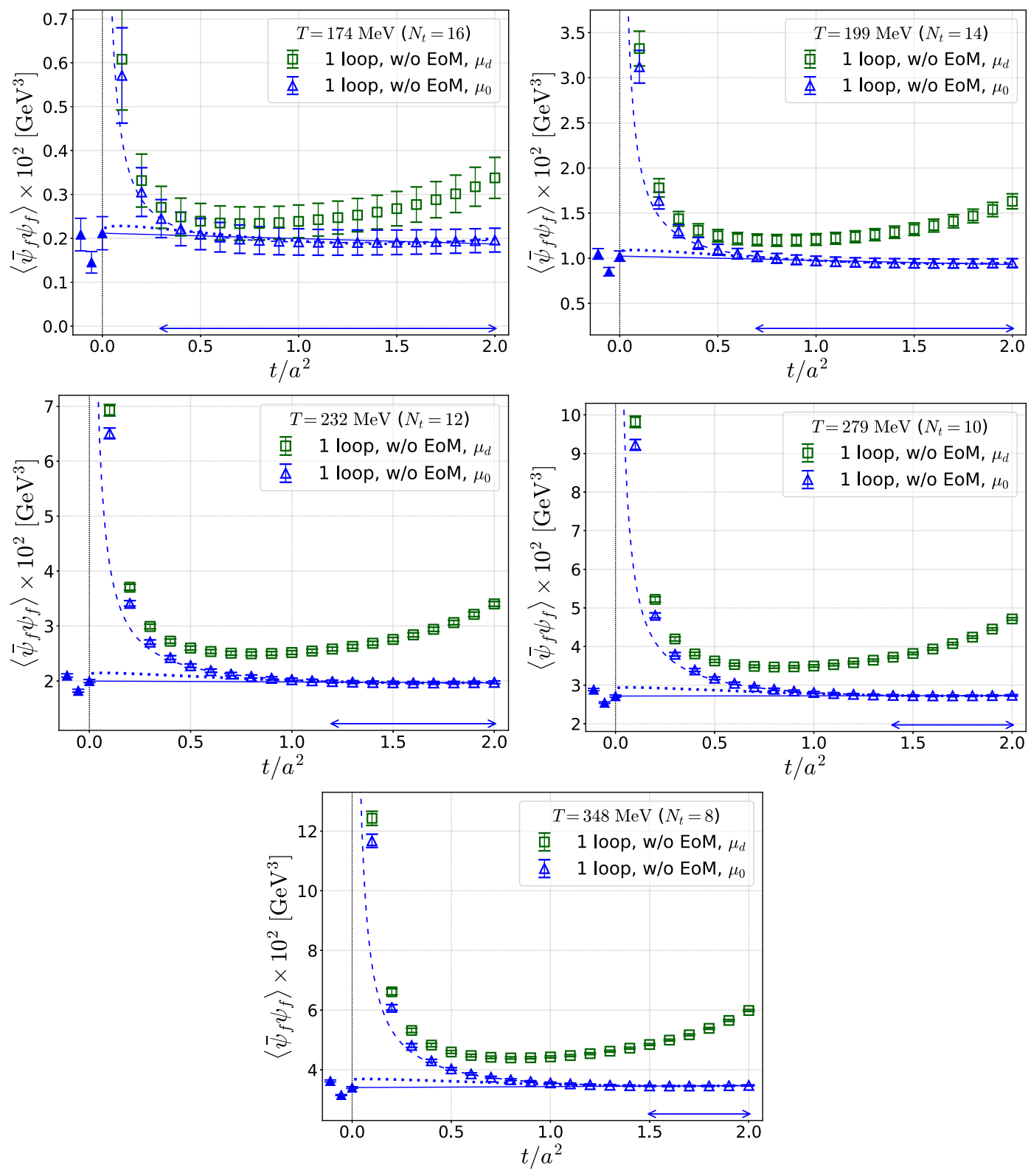

FIG. 4. The same as Fig. 2 but for the chiral condensate $\left\langle\left\{\bar{\psi}_{f} \psi_{f}\right\}\right\rangle$ for $f=u$ or $d$ with VEV subtraction. The vertical axis is in units of $\mathrm{GeV}^{3}$. 

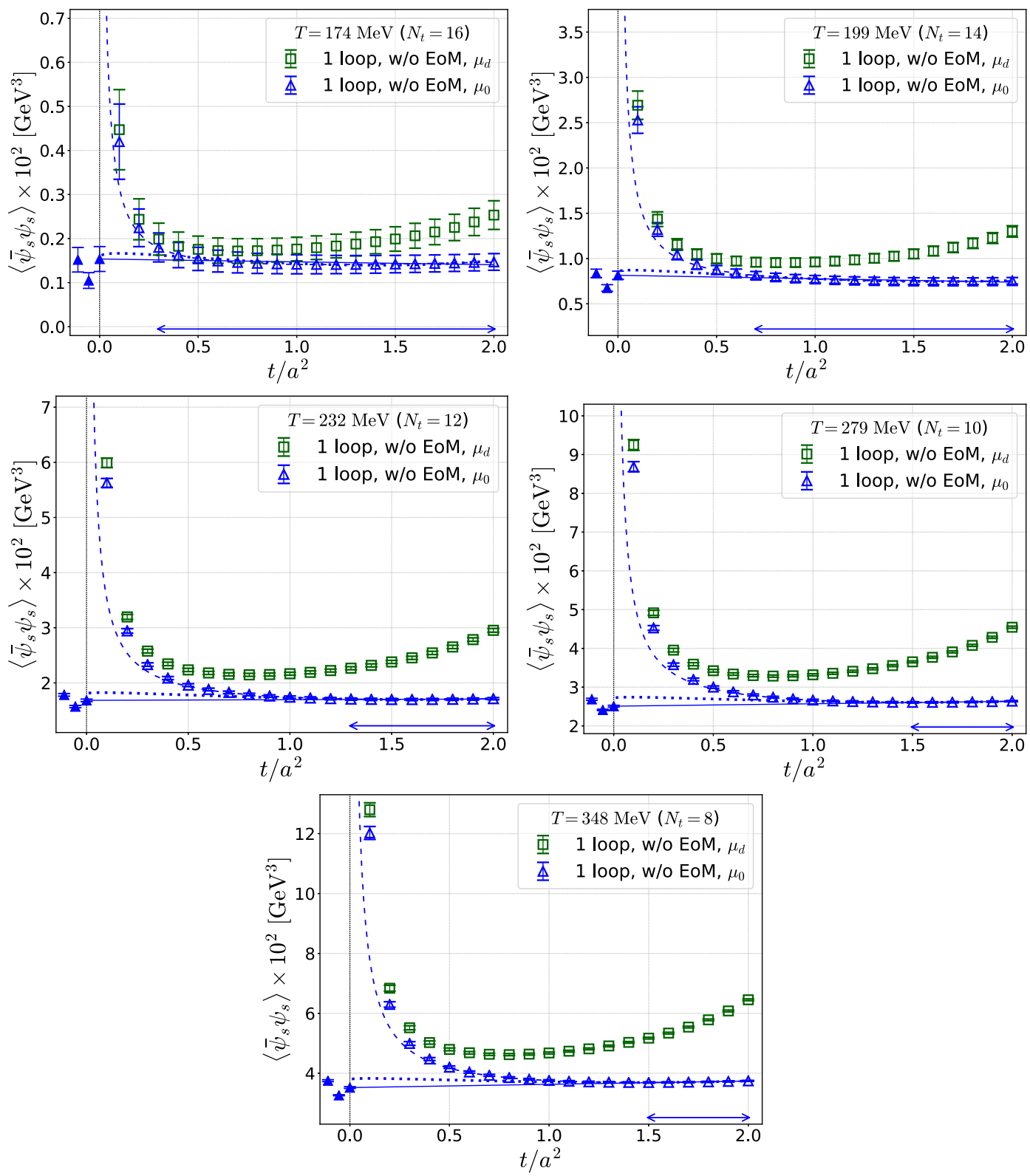

FIG. 5. The same as Fig. 4 but for the $s$ quark $(f=s)$. 

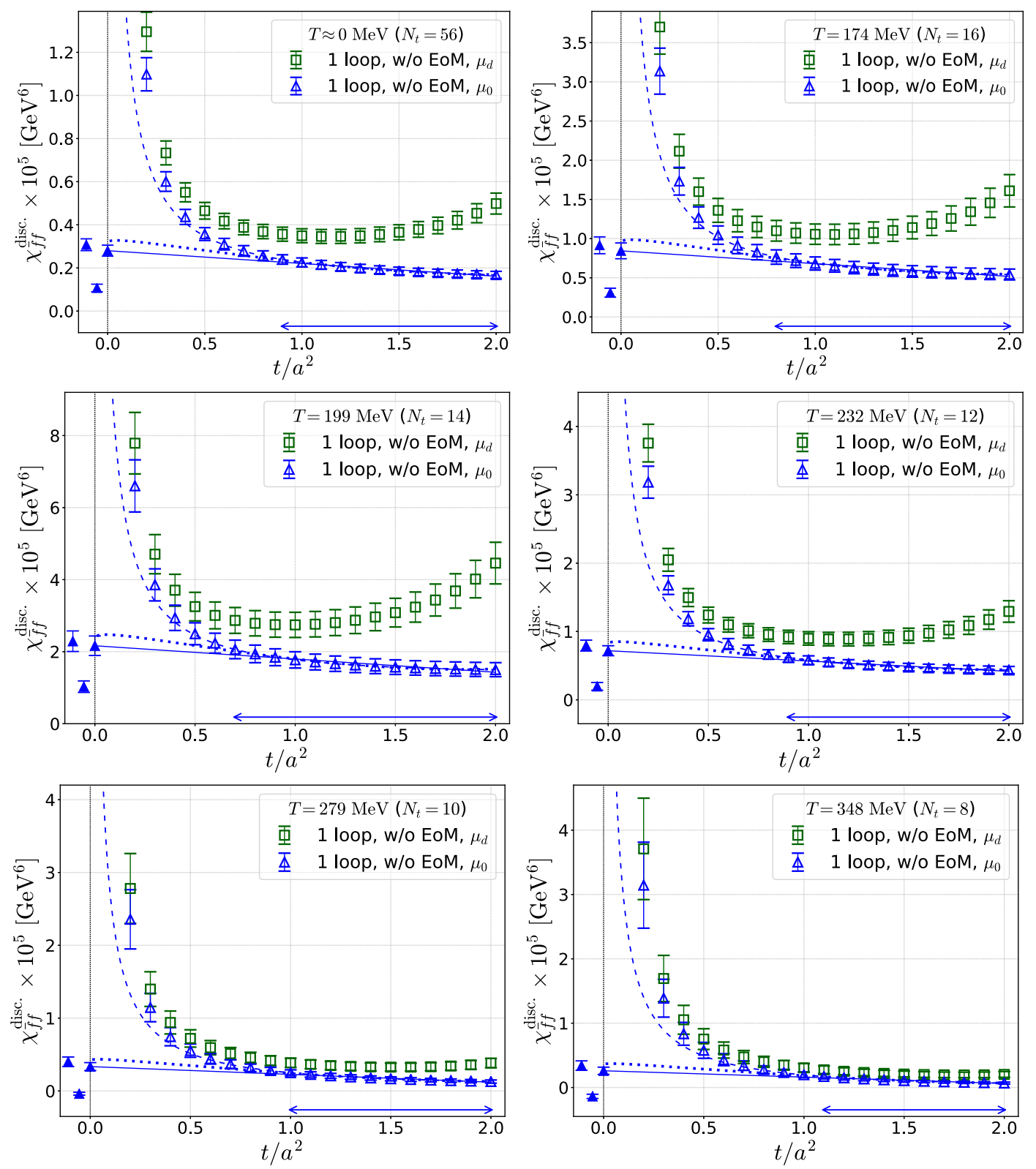

FIG. 6. The same as Fig. 2 but for the disconnected chiral susceptibility $\chi_{\overline{f f}}^{\text {disc }}$ in the $\overline{\mathrm{MS}}$ scheme at $2 \mathrm{GeV}$ for $f=u$ or $d$ quark. The vertical axis is in units of $\mathrm{GeV}^{6}$. 

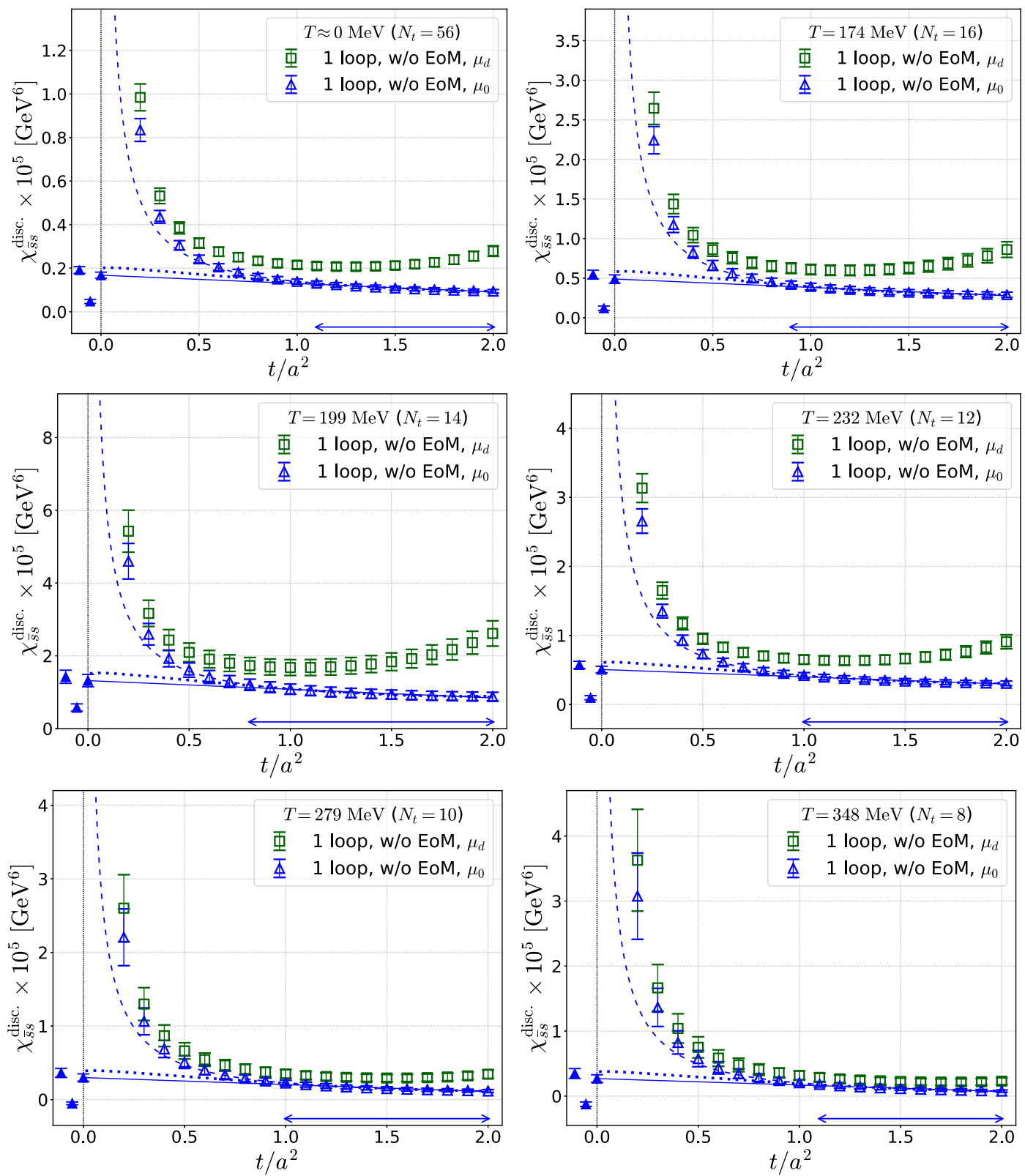

FIG. 7. The same as Fig. 6 but for the $s$ quark. 

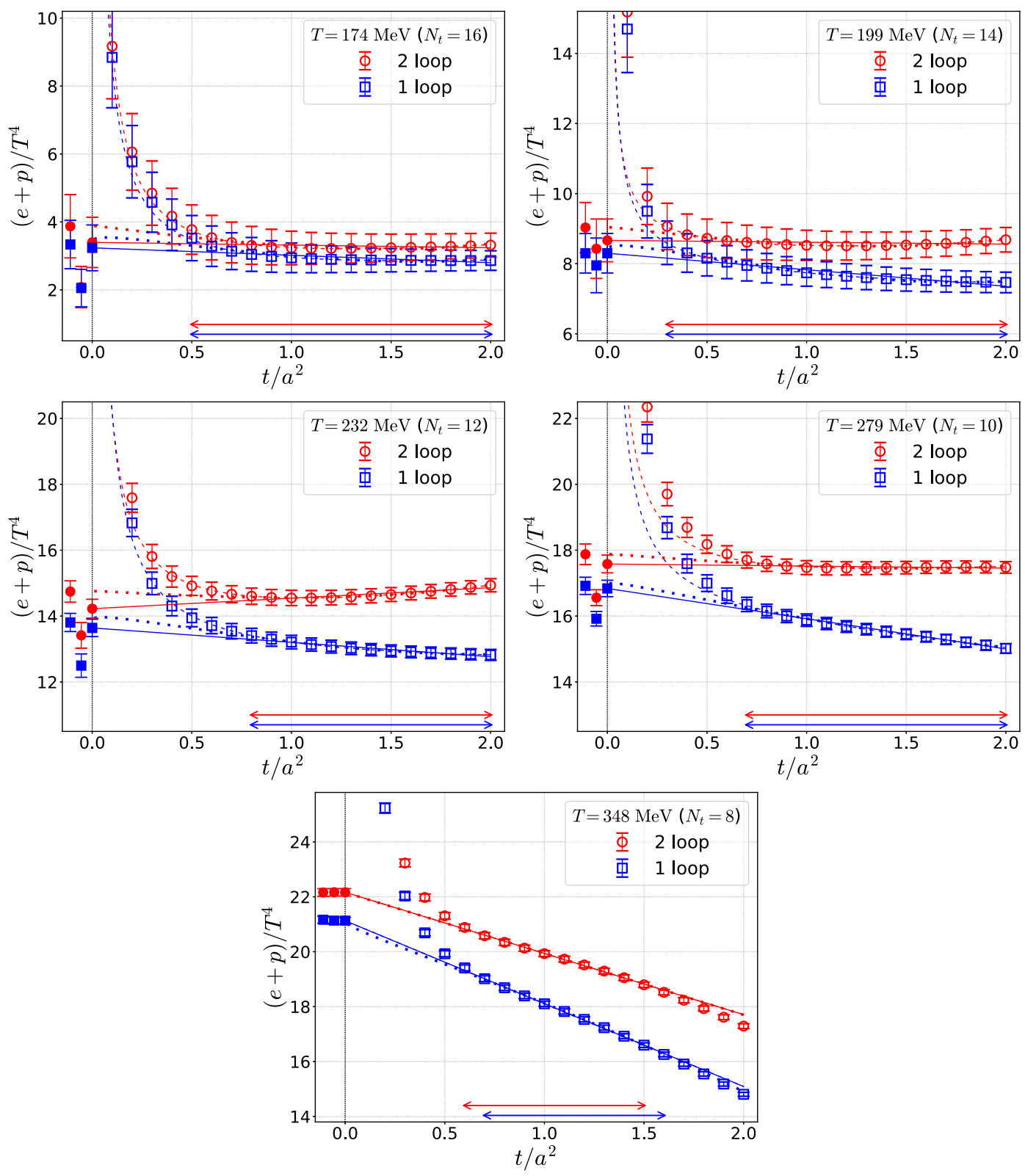

FIG. 10. Entropy density $(\epsilon+p) / T^{4}$ with the $\mu_{0}$ scale as a function of the flow time. Results using one-loop matching coefficients of Ref. [10] are compared with those using two-loop matching coefficients of Ref. [35]. The arrows at the bottom indicate the linear windows at each temperature. Symbols at $t / a^{2}=0$ and solid lines with the same color are the results of the linear fits. Fit results with the nonlinear ansatz (49) and linear $+\log$ ansatz (50) or (51) are shown by dashed and dotted curves together with the symbols at $t / a^{2}<0$ to the right and to the left, respectively. Errors are statistical only. 

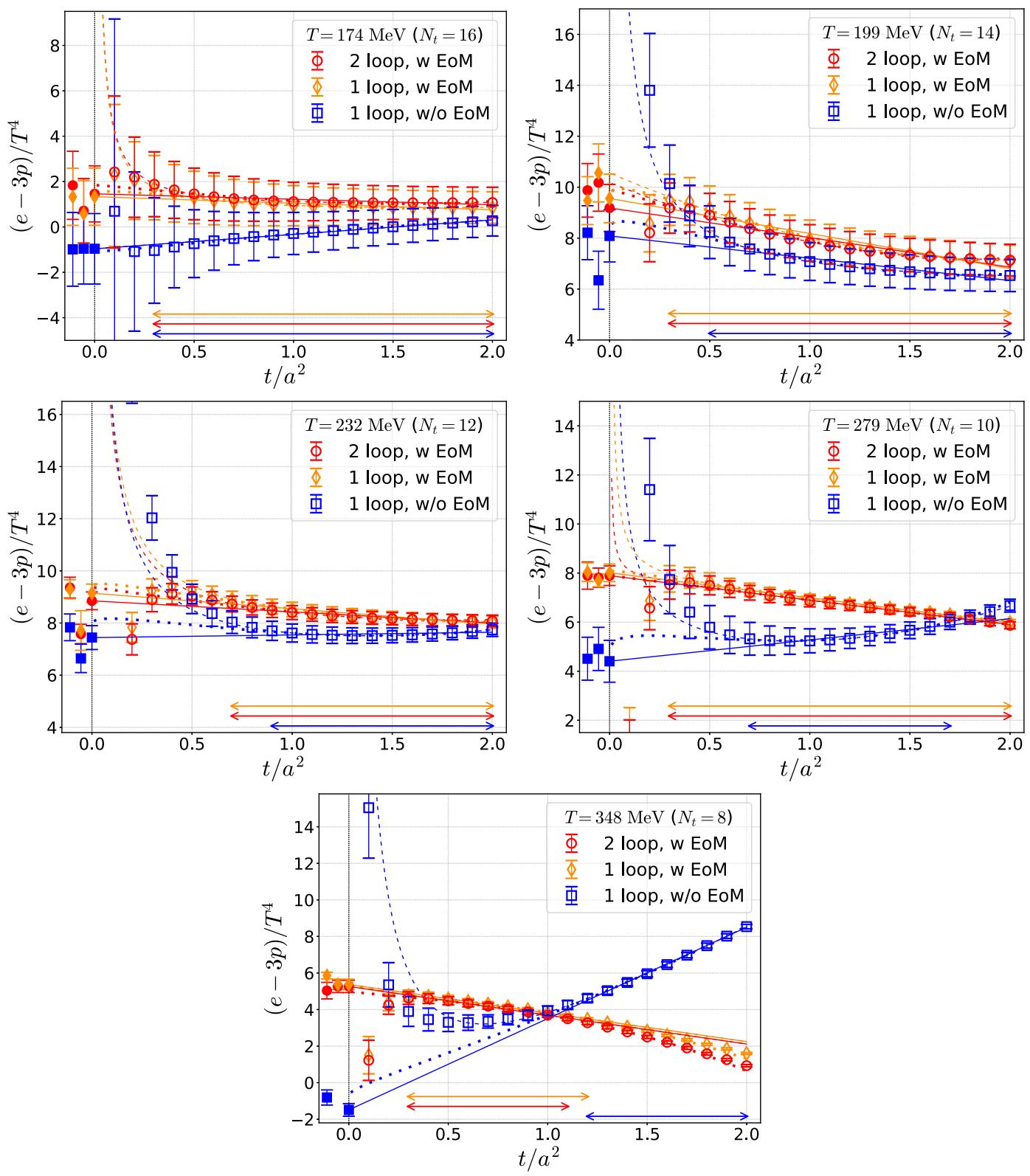

FIG. 12. The same as Fig. 10 but for the trace anomaly $(\epsilon-3 p) / T^{4}$. Also shown are the results using the one-loop part of the matching coefficients of Ref. [35] in which the EoM is used.

[1] M. Tanabashi et al. (Particle Data Group), Phys. Rev. D 98, 030001 (2018). 